\title{
Evidence for the occurrence of a prototype structure in Sc under pressure
}

\author{
Y. C. Zhao, ${ }^{*}$ F. Porsch, and W. B. Holzapfel \\ Fachbereich Physik, Universität-GH-Paderborn, D-33095 Paderborn, Germany
}

(Received 17 November 1995; revised manuscript received 11 April 1996)

\begin{abstract}
Structures and pressure-volume relations of scandium under pressures up to $76 \mathrm{GPa}$ have been studied at room temperature with diamond-anvil cells by energy-dispersive $\mathrm{x}$-ray diffraction using synchrotron radiation. In contrast to previous studies, many more diffraction lines are recorded for $\mathrm{Sc}(\mathrm{II})$, stable from 17.2 to at least $76 \mathrm{GPa}$, the highest pressure of the present study. A pseudo-body-centered-cubic lattice with 24 atoms in the cubic cell results in a perfect indexing of all the diffraction lines observed and some information on the atomic arrangement is derived from the intensity pattern. Since no other element shows the same kind of diffraction pattern, this phase represents a new prototype structure, and Sc under pressure follows a special structural sequence with significant differences to the systematics observed in most of the other (trivalent) rare-earth metals. [S0163-1829(96)00738-2]
\end{abstract}

\section{INTRODUCTION}

Scandium with its one $3 d$ electron in the outer shell of its free atoms represents the first member of the $d$-transitionmetal group. Conventionally, Sc, Y and the lanthanide metals $(L)$ are grouped together as the rare-earth $(\mathrm{RE})$ metals $^{1}$ due to their similarities in their outer electron configurations and in many of their physical and chemical properties. However, in contrast to Sc, the trivalent $L$ metals and Y undergo under pressure a common sequence of structural transitions in the direction $^{2-5} \quad \mathrm{hcp}(h P 2) \rightarrow \operatorname{Sm}$-type $(h R 9)$ $\rightarrow \operatorname{dhcp}(h P 4) \rightarrow \mathrm{fcc}(c F 4) \rightarrow$ distorted-fcc $(h R 24$ ? $)$. Thereby the symbols in parentheses represent Pearson's notation, which is strongly recommended for more systematic use by International Union of Pure and Applied Chemistry (IUPAC). ${ }^{6}$ At ambient pressure Sc crystallizes also in the $h P 2$ structure and this phase is commonly denoted as $\mathrm{Sc}(\mathrm{I})$. The phase transition to Sc(II) was observed around $19 \mathrm{GPa}$ first by superconductivity and by room-temperature resistance measurements, ${ }^{7}$ and later confirmed by x-raydiffraction measurements, ${ }^{8,9}$ which indicated that $\mathrm{Sc}(\mathrm{II})$ does not belong to any of the regular RE structures, but might be characterized possibly by a primitive tetragonal lattice. ${ }^{8,9}$ Moreover, by correlating the structural energies with the $d$-band occupancy, a first-principles calculation ${ }^{10}$ resulted in the following structural sequence for $\mathrm{Sc}$ with increasing pressures: $h P 2-c F 4-h P 3$ ( $\omega$-phase)- $t P 4-c I 2$. Thereby the calculated atomic volumes at the $h P 2-c F 4$ and $c F 4-h P 3$ transitions were both close to the value observed experimentally for the $\mathrm{Sc}(\mathrm{I}) \rightarrow \mathrm{Sc}$ (II) transition. Recently, a close resemblance in the phonon dispersion was observed in the hightemperature $c I 2$ phases of Sc with respect to its neighboring group IVB metals, and therefore it was speculated ${ }^{11}$ that the $h P 2-h P 3$ transition $^{12}$ found in the group IVB metals may also occur in Sc under pressure. However, according to the previous X-ray-diffraction studies ${ }^{8,9}$ the structure of $\mathrm{Sc}$ (II) is distinctly different from the $h P 3$ structure. Since the previous structural proposals were based only on measurements with conventional $x$-ray sources with contradictory interpretations of the poorly resolved patterns ${ }^{8,9}$ and also in contrast with the theoretical predictions, ${ }^{10}$ it appeared appropriate to use synchrotron radiation to obtain $\mathrm{x}$-ray-diffraction patterns for $\mathrm{Sc}$ (II) with higher resolution to resolve its structure.

\section{EXPERIMENTAL}

The present experiments were performed with synchrotron radiation at HASYLAB, Hamburg, at the station for energy-dispersive $\mathrm{X}$-ray-diffraction (EDXD) as described previously. ${ }^{13,14}$ Pressures were generated with diamond-anvil cells $^{15,16}$ and inconel gaskets together with liquid nitrogen or mineral oil as pressure transmitting medium. Ruby powder served as a sensor for the pressure determination from the shift of the $R_{1}$ ruby luminescence line according to the nonlinear pressure scale. ${ }^{17}$ Seven Sc samples were studied in order to test the reproducibility of the experimental results. Most of the experiments were performed with a diffraction angle $2 \theta$ around $10^{\circ}$, some others with $2 \theta=5.644^{\circ}$, corresponding to $E d=12593.1 \mathrm{keV} \mathrm{pm}$, allowed us to recover also diffraction peaks with lattice spacings $d_{h k l}$ up to 630 pm. The sample material was provided by K. A. Gschneidner, Jr., with chemical analysis indicating as major impurities in atomic ppm 294 for hydrogen, 306 for oxygen, 29 for iron, and 80 for lanthanides.

\section{RESULTS AND DISCUSSION}

$h P 2-\mathrm{Sc}(\mathrm{I})$ is observed up to about $20 \mathrm{GPa}$ in all the experiments but first signs of $\mathrm{Sc}(\mathrm{II})$ appear at $17.2 \mathrm{GPa}$, as shown in Fig. 1. Sc(II) remains stable at least up to $76 \mathrm{GPa}$, the highest pressure of the present study. Figure 2 shows EDXD spectra for $\mathrm{Sc}(\mathrm{II})$ at $31.0 \mathrm{GPa}$. The present diffraction spectra with their higher resolution show first of all that a large body-centered-cubic unit cell with $a=741.2(1) \mathrm{pm}$ allows us to index all the observed peaks as shown in Fig. 3 and in Table I for a spectrum of Sc(II) taken at 31.0 GPa. This indexing was performed with the special software XPOWDER, ${ }^{18}$ which searched for all possible structures within the cubic, tetragonal, orthorhombic, rhombohedral, and hexagonal systems, excluding only the monoclinic and triclinic systems due to their extremely large number of possibilities and very low likeliness for such a simple diffraction pattern, 


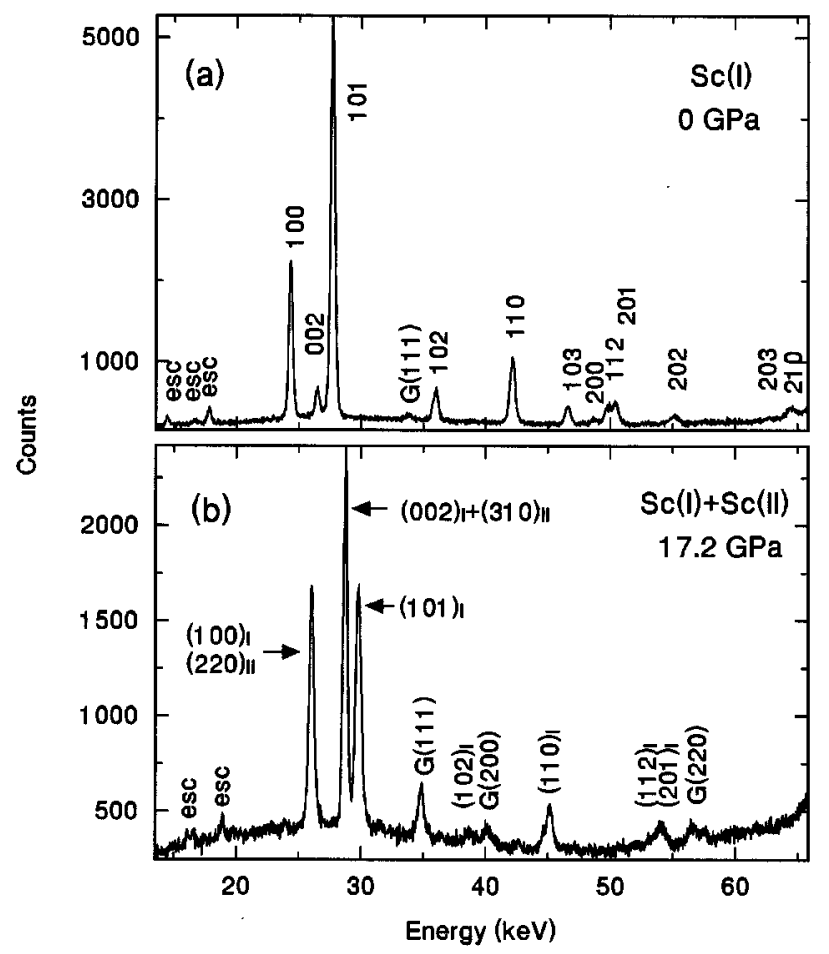

FIG. 1. EDXD spectra for $h P 2-\mathrm{Sc}(\mathrm{I})$ at ambient pressure (a) and for the mixture of $\mathrm{Sc}(\mathrm{I})$ and $\mathrm{Sc}(\mathrm{II})$ at $17.2 \mathrm{GPa}$ (b) taken with $E d=6981.6 \mathrm{keV} \mathrm{pm}$ corresponding to $2 \theta=10.188^{\circ}$. Escape peaks and diffraction peaks from the inconel gasket are denoted by esc and $G$, respectively.

allowing for unit-cell volumes up to $1 \mathrm{~nm}^{3}$. This program resulted in only this solution or trivial equivalent indexing and all the observed lines are indexed by this scheme with a standard error of $\sigma=0.12 \%$ with $\sigma=\left\{\Sigma\left[\left(d_{\mathrm{obs}}-d_{\mathrm{cal}}\right) /\right.\right.$ $\left.\left.d_{\mathrm{obs}}\right]^{2} /(N-1)\right\}^{1 / 2}$. This standard deviation fits to the typical experimental accuracy, and only three expected peaks in the experimental range down to $110 \mathrm{pm}$ are not observed within the present sensitivity, recovering intensities down to $0.1 \%$ with respect to the largest intensity observed for the (310) peak. Only the peaks with intensities larger than $1.6 \%$ were observed in the previous studies ${ }^{8,9}$ as shown in Fig. 3, where the present data are denoted by solid dots and previous data ${ }^{9}$ by open circles. The previous proposals of different $t P 4$ structures for the lattice of $\mathrm{Sc}(\mathrm{II})$ result in significantly larger values for $\sigma(>0.5 \%)$, and do not reproduce most of the weaker peaks discovered in the present measurements. Therefore, the previous assignments, ${ }^{8,9}$ with two different primitive tetragonal lattices can be ruled out whereas the present assignment of a bcc lattice for $\mathrm{Sc}$ (II) seems to result in a perfect fit.

Since the atomic volume for $\mathrm{Sc}(\mathrm{I})$ at the phase transition sets a lower limit to the number of atoms in the proposed unit cell for $\mathrm{Sc}(\mathrm{II})$, the most reasonable result is obtained for 24 atoms per unit cell, which leads to a volume decrease of $6.5 \%$ at the phase transition from $\mathrm{Sc}(\mathrm{I})$ to $\mathrm{Sc}(\mathrm{II})$, whereas 26 atoms in this unit cell would result in a rather large volume decrease of $13.7 \%$. In Pearson's notation $c I 24$ appears at this stage as the most likely structure and $c I 26$ remains as an other possible candidate. Since none of the elements is known to crystallize in one of these structures at any
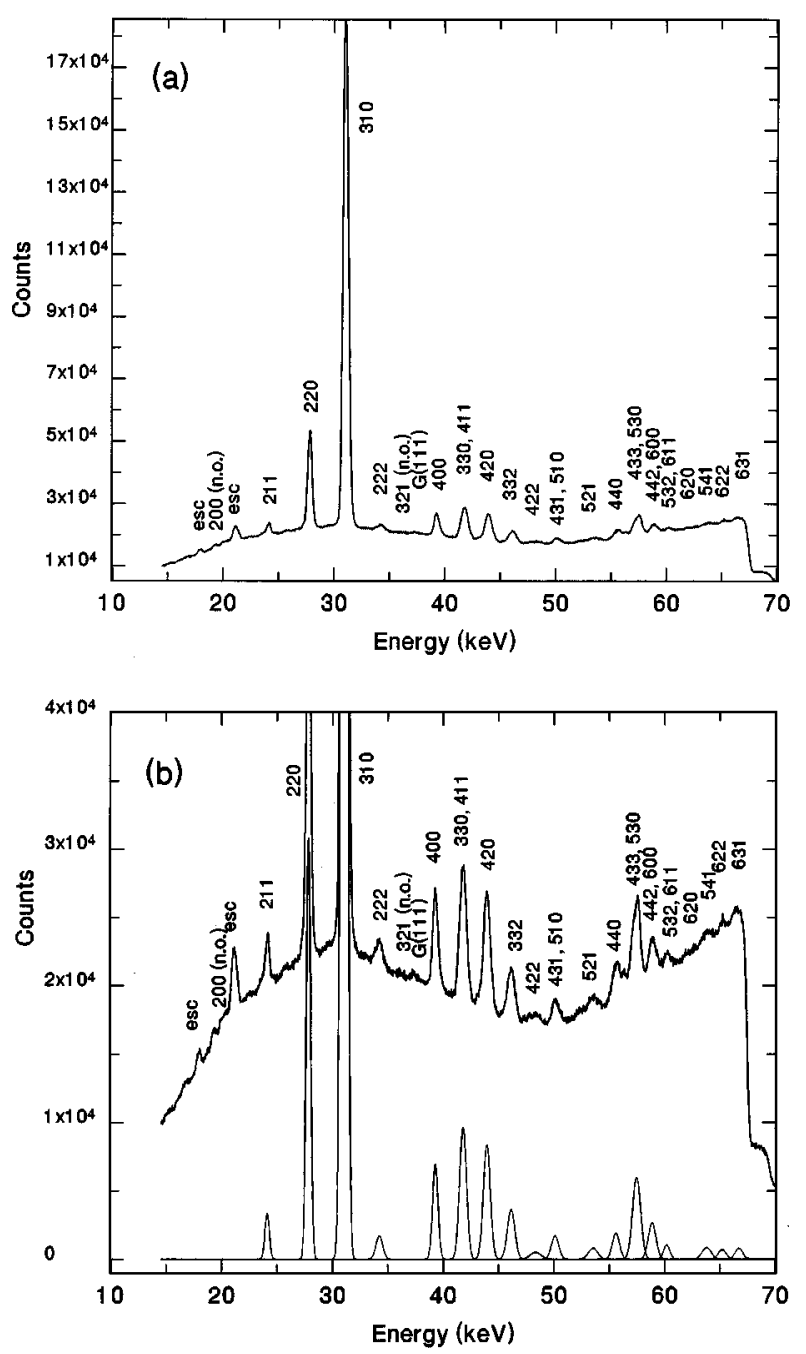

FIG. 2. EDXD spectrum for $\mathrm{SC}(\mathrm{II})$ at $31.0 \mathrm{GPa}$ with $E d$ $=7289.1 \mathrm{keV} \mathrm{pm}$ corresponding to $2 \theta=9.575^{\circ}$. The spectrum is indexed according to a bcc lattice with $a=741.2(1) \mathrm{pm}$. Escape peaks, diffraction peaks from the gasket, and the not observed peaks are denoted by esc, $G$, and n.o., respectively. The result of fitting Gaussian profiles to the observed diffraction peaks is shown in the lower part of (b).

condition, ${ }^{4,19-23} \mathrm{Sc}$ (II) presents a new prototype structure. In order to locate the atoms in this bcc unit cell, diffraction intensities were calculated and then compared with the observed diffraction intensities. There are nine space groups with a bcc lattice but only two of them, $I 23$ and $I 2{ }_{1} 3$, need to be considered for these calculations, because all others are supergroups of these two. However, with full variation of the free atomic positional parameters for all possible combinations of site occupations for these two space groups, none of the calculated spectra agreed with the observed intensity data within the experimental uncertainty allowing also for some texture. This observation leads to a dilemma: an excellent agreement between observed and calculated $d$ values and a poor agreement between observed and calculated intensities. To solve this dilemma one could assume that extremely strong but reproducible texture falsifies the observed intensity data or otherwise that the lattice of Sc(II) shows only some similarity to the proposed body-centered-cubic lattice 


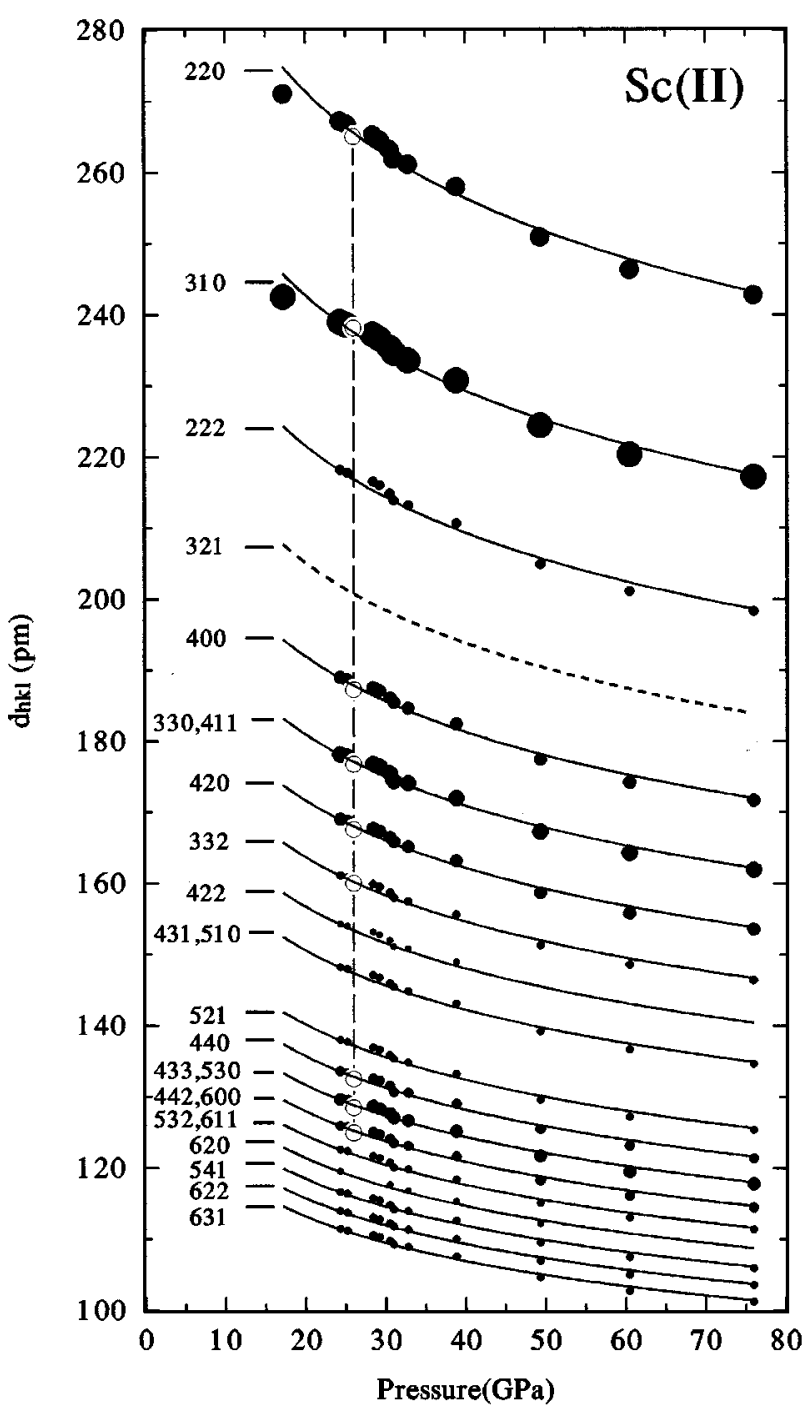

FIG. 3. $d_{h k l}$ data for $\mathrm{Sc}(\mathrm{II})$ with the present indexing scheme. The present data are represented by solid circles with their sizes denoting the intensities of the corresponding diffraction peaks. The only set of data at $26.0 \mathrm{GPa}$ given in Ref. 9 is represented by open circles.

but the actual symmetry of $\mathrm{Sc}(\mathrm{II})$ is lower than $I 23$ or $I 2{ }_{1} 3$. The first case appears to be unlikely because all the present experiments show reasonably reproducible relative intensities which agree also qualitatively with the intensities observed in the earlier experiments. ${ }^{8,9}$ Therefore, a pseudocI24 (or pseudo-cI26) structure fits best to the present data for $\mathrm{Sc}(\mathrm{II})$.

In order to make a more detailed structural proposal for $\mathrm{Sc}(\mathrm{II})$, one can start with the lowest symmetry space group $P 1$ with the parameters of the triclinic unit cell $a=b=c=(\sqrt{3 / 2}) a_{\mathrm{bcc}}$ and $\alpha=\beta=\gamma=109^{\circ} 28^{\prime}$, where $a_{\mathrm{bcc}}$ is the unit-cell parameter of the bcc lattice, with the assignment of 12 or 13 atoms to the $1 a$ position, and then determine $12 \times 3$ or $13 \times 3$ free parameters for the atomic positions. On the other hand, the structure of $\mathrm{Sc}$ (II) may be modeled by packing one structural unit in the lattice under the constraints of the combination of the point symmetry for the structural unit and the transitional symmetry for the lattice. For this
TABLE I. Measured intensities $I_{\text {obs }}$ and lattice spacings $d_{\text {obs }}$ for $\mathrm{Sc}$ (II) at $31.0 \mathrm{GPa}$ in comparison with calculated values $d_{\text {cal }}$ for a bcc unit cell with $a=741.2(1) \mathrm{pm}$. Miller indices $h k l$ and the relative deviations $\Delta d / d$ of the fitted $d$ values are also presented. $M$ denotes the number of the $\{h k l\}$ faces with the same $d$ spacing. n.o. denotes not observed peaks.

\begin{tabular}{|c|c|c|c|c|c|}
\hline$h k l$ & $I_{\mathrm{obs}}$ & $\begin{array}{l}d_{\mathrm{obs}} \\
(\mathrm{pm})\end{array}$ & $\begin{array}{l}d_{\mathrm{cal}} \\
(\mathrm{pm})\end{array}$ & $\begin{array}{c}\left(d_{\mathrm{obs}^{-}}\right. \\
\left.d_{\mathrm{cal}}\right) / d_{\mathrm{obs}}(\%)\end{array}$ & $M$ \\
\hline 110 & n.o. & n.o. & 524.1 & & 12 \\
\hline 200 & n.o. & n.o. & 370.6 & & 6 \\
\hline 211 & 1.7 & 302.1 & 302.6 & -0.17 & 24 \\
\hline 220 & 15.6 & 261.9 & 262.0 & -0.04 & 12 \\
\hline 310 & 100 & 234.7 & 234.4 & 0.13 & 24 \\
\hline 222 & 1.4 & 213.9 & 214.0 & -0.05 & 8 \\
\hline 321 & n.o. & n.o. & 198.1 & & 48 \\
\hline 400 & 4.3 & 185.5 & 185.3 & 0.11 & 6 \\
\hline 330,411 & 7.7 & 174.4 & 174.7 & 0.00 & 12,24 \\
\hline 420 & 6.8 & 165.9 & 165.7 & 0.12 & 24 \\
\hline 332 & 3.2 & 158.0 & 158.0 & 0.00 & 24 \\
\hline 422 & 0.6 & 151.1 & 151.3 & -0.13 & 36 \\
\hline 431,510 & 1.5 & 145.5 & 145.4 & 0.07 & 48,24 \\
\hline 521 & 0.9 & 135.4 & 135.5 & 0.07 & 48 \\
\hline 440 & 1.6 & 130.7 & 131.0 & -0.23 & 12 \\
\hline 433,530 & 5.6 & 126.9 & 127.1 & -0.16 & 24,24 \\
\hline 442,600 & 2.4 & 123.7 & 123.5 & 0.16 & 24,6 \\
\hline 532,611 & 0.8 & 120.1 & 120.2 & -0.08 & 48,24 \\
\hline $620^{\mathrm{a}}$ & $<0.1$ & 117.0 & 117.2 & -0.17 & 24 \\
\hline 541 & 0.6 & 114.2 & 114.4 & -0.18 & 48 \\
\hline 622 & 0.4 & 111.8 & 111.7 & 0.09 & 24 \\
\hline 631 & 0.4 & 109.2 & 109.3 & -0.09 & 48 \\
\hline
\end{tabular}

${ }^{\mathrm{a}}$ This peak has only marginal intensity.

approach, some special features of the spectra for $\mathrm{Sc}$ (II) should be noted: The (110) and (200) peaks have never been observed in any of the diffraction spectra with small diffraction angles, which extend into the appropriate range covering the $d$ values of the (110) and (200) peaks: The absence of these two peaks cannot be attributed to texture because the corresponding higher-order reflections (220) and (400) are very well observed. The (321) peak with its large multiplicity factor has never been observed, and furthermore, the diffraction intensities seem to be modulated, suggestive of a specific cluster stacking in the structure of $\mathrm{Sc}(\mathrm{II})$. An icosahedral cluster, which consists of 12 atoms at the vertices and 1 atom at the center, is one of the most stable and densest packings, and possible spontaneous distortions of the cubooctahedral atomic clusters of $c F 4$ or $h P 3$ into icosahedral clusters have been discussed before from the point of view of local energetics. ${ }^{24}$ Stimulated by this approach, one may consider that the structure of $\mathrm{Sc}$ (II) could be built up by ideal or distorted icosahedral clusters. Since the diffraction intensities are proportional to the square of the total scattering factor $F_{\text {total }}$, the present model would result in $F_{\text {total }}$ $=F_{\text {lattice }} \times F_{\text {cluster }}$, where $F_{\text {lattice }}$ and $F_{\text {cluster }}$ are the form factors for the bcc lattice and the atomic cluster, respectively. $F_{\text {lattice }}=2$ for every $(h k l)$ of the bcc lattice and $F_{\text {cluster }}$ $=\int \rho(r) \exp (i k \cdot r) d^{3} r$, where $\rho(r)$ is the electron density for the atomic cluster. Without any further knowledge, $\rho(r)$ can be expressed as a multipole expansion in spherical polar 


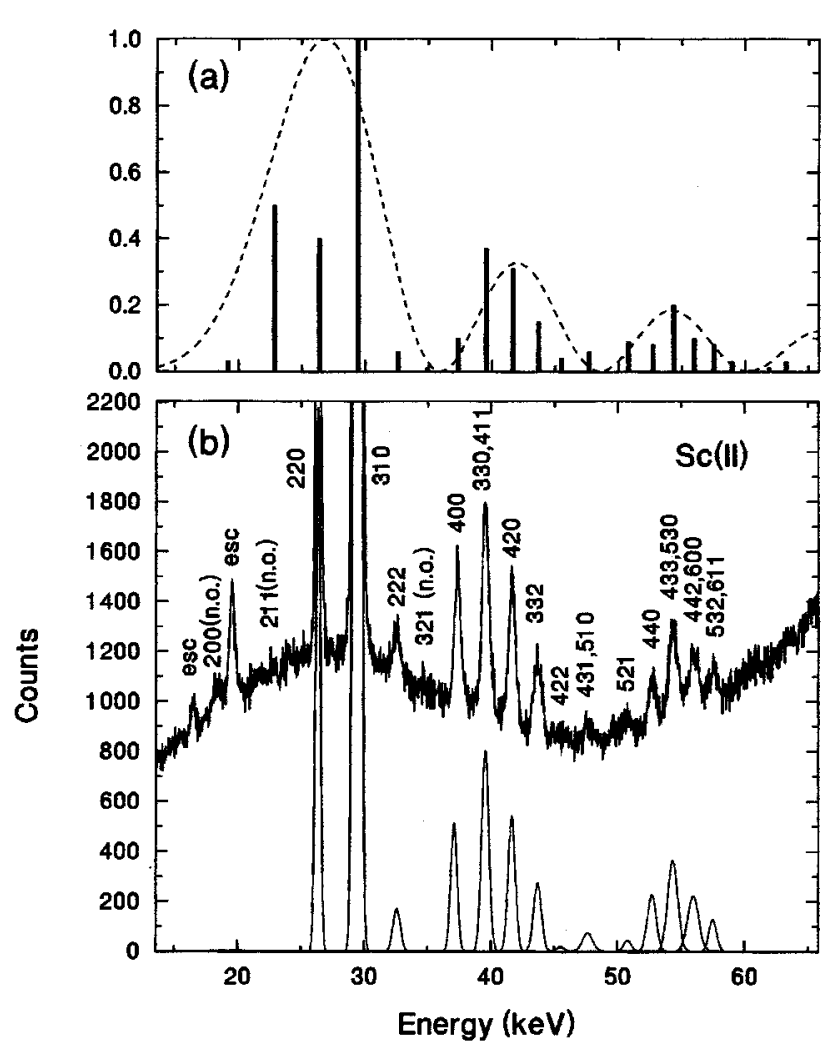

FIG. 4. Comparison of the model calculation for the intensities with an EDXD spectrum for $\mathrm{Sc}$ (II) at $29.2 \mathrm{GPa}$ taken with $E d$ $=6981 \mathrm{keV}$ pm corresponding to $2 \theta=10.188^{\circ}$. The spectrum is indexed to a bcc lattice with $a=748.5(2) \mathrm{pm}$. The variation of the relative intensities with respect to Fig. 2 indicates some effects of texture, most noticeable for (211). (a) illustrates by the dashed curve the variation of $\left[j_{6}(1.014 E R \sin \theta)\right]^{2}$ for $R=325 \mathrm{pm}$ together with calculated intensities by vertical solid bars according to the present model. The result of fitting Gaussian functions to the observed diffraction peaks is shown in the lower part of (b).

coordinates $^{25}$ and $\exp (i k \cdot r)$ can be expanded ${ }^{25}$ in terms of spherical Bessel functions $j_{l}(k r)$ and harmonic functions $Y_{l m}(\theta, \phi)$. For the icosahedral cluster only the terms with $l=6$ dominate. ${ }^{26}$ If the radial distribution of the electron density is approximated by a $\delta$ function with radius $R$ corresponding to the size of the cluster, one arrives then at $F_{\text {cluster }} \propto j_{6}(k R)$. For the present EDXD method, the momentum transfer of photons scattered by the cluster is $k=4 \pi \sin \theta / \lambda=1.014 /$ $(\mathrm{pm} \mathrm{keV)} E \sin \theta$, where $E$ is the photon energy. The values of $\left[j_{6}(k R)\right]^{2}$ with $R=325 \mathrm{pm}$ are shown by the dashed line in Fig. 4(a), and Fig. 4(b) presents a diffraction pattern observed at $29.2 \mathrm{GPa}$ for comparison. An atom located at $R=0$ would contribute to the term with $l=0$, however, having only the scattering power of $(1 / 13)^{2}$ with respect to the 12 remaining atoms of the cluster. For a real icosahedral cluster, $F_{\text {cluster }}$ would depend also on the orientation of the clusters, represented by the values of $Y_{l m}(\theta, \phi)$. However, the integrated diffraction intensity results from all the lattice plans with the same $d$ spacing averaged over all crystallites within the $\mathrm{x}$-ray-irradiated region of the sample. The number of differently oriented planes with the same spacing is usually rather large as shown in Table I by the multiplicity $M$. Thus, if texture can be neglected, the orientation dependence of
$F_{\text {cluster }}$ may be averaged out largely and absorbed in the photon energy-independent prefactor in the formula for the calculation of integrated intensities. ${ }^{27}$ The corresponding model calculations for such a simplified cluster scattering shows in Fig. 4 indeed some resemblance to the observed EDXD spectra for $\mathrm{Sc}$ (II), when the appropriate values for $M, F_{\text {lattice }}$, and $F_{\text {cluster }}$ are used in the expression for the integrated intensities. The most obvious discrepancy between these calculated and observed intensities is then noticed for the (211) peak. This deviation could possibly be due to texture as indicated by the difference between the data illustrated in Figs. 1 and 2.

If one tries to work out a model of icosahedral-cluster packing, one finds that the detailed structure would depend on the orientation of the cluster with respect to the crystal axes. Without any knowledge of constraints upon the orientations of the cluster imposed by the site symmetries of the atoms, one can consider the constraints due to the cluster radius $R=325 \mathrm{pm}$ related to the modulations in Fig. 4(a). Since $2 R=650 \mathrm{pm}$ corresponds to almost half of the body diagonal of the pseudo-bcc lattice $(\sqrt{3 / 2}) a_{\mathrm{bcc}}=648 \mathrm{pm}$ at 29.2 GPa, the clusters would have to be linked by sharing common vertices along one of the body diagonals of the bcc unit cell. However, due to the symmetry of the icosahedron these arrays should not be linked by other common vertices. With this kind of linking, each pseudocubic lattice site would be occupied by 12 atoms, corresponding to a pseudo-cI 24 structure for $\mathrm{Sc}(\mathrm{II})$. This pseudo-cI24 structural proposal or some similar quasicrystalline structure are furthermore supported by the observation that this structure would result for the volume decrease in the transition of $\mathrm{Sc}$ (I) to $\mathrm{Sc}$ (II) (as mentioned before) in a more reasonable value than the pseudo-cI26 structure, with its 13 atoms on each lattice site. Therefore, at this stage a pseudo- $c I 24$ lattice is regarded as the most reasonable candidate for the structure of $\mathrm{Sc}$ (II).

With this structural model, the pressure dependence of the atomic volume can be calculated also for this phase to allow for a comparison with the data for $\mathrm{Sc}(\mathrm{I})$ and with some previous results for $\mathrm{Sc}(\mathrm{II}){ }^{28,29}$ as shown in Fig. 5. The present data for $\mathrm{Sc}(\mathrm{I})$ are consistent with the previous data, which can be represented by any of the common equation-of-state (EOS) forms using ambient pressure values for the atomic $V_{0}=0.02499 \mathrm{~nm}^{3}$, for the bulk modulus $K_{0}=60(1) \mathrm{GPa}$ and for its pressure derivative $K_{0}^{\prime}=2.8(3)$. The small difference between the previous and the present data for the $\mathrm{Sc}$ (II) is partly caused by the different structural assignments, and the first data point in the transition region is considered to be less accurately determined due to phase mixture and the limited number of lines recorded in this case as shown in Fig. 3. Since the measured data cluster strongly around $30 \mathrm{GPa}$, five times stronger weight was given to the last two data at 60 and $76 \mathrm{GPa}$ to obtain more reasonable results in the leastsquares fitting of an analytic form to these data. Due to the small curvature in these data the common practice of using an EOS form with the three free parameters $V_{0}, K_{0}$, and $K_{0}^{\prime}$ for the volume, bulk modulus, and its pressure derivative extrapolated to zero pressure leads to large correlated uncertainties in these parameters for any of the common secondorder EOS forms. This large ambiguity can be avoided to some extend, if one uses at first the convenient second-order form previously denoted $^{30} \mathrm{H} 02$ : 


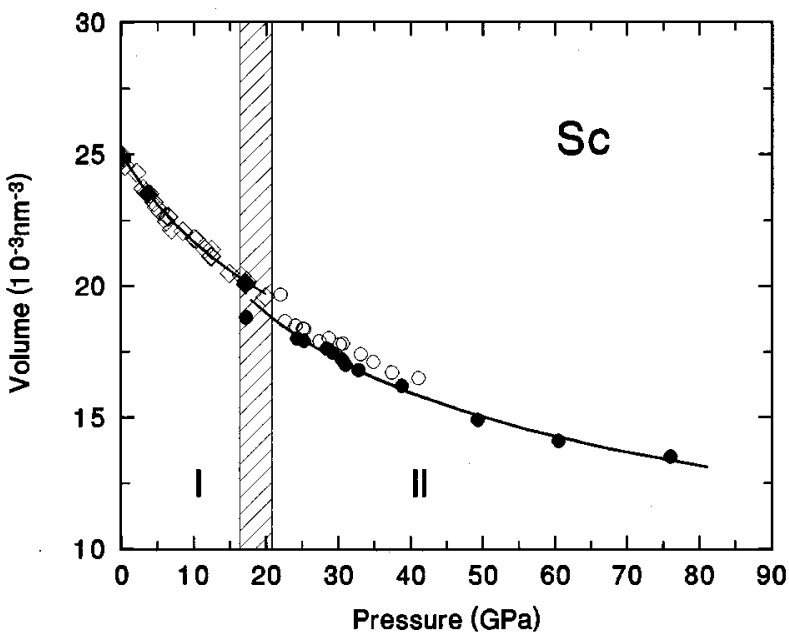

FIG. 5. Pressure-volume relation of Sc. Open symbols represent previous data (Refs. 29 and 30) and the solid symbols denote the present results with the assignment of the pseudocubic lattice $c I 24$ to $\mathrm{Sc}(\mathrm{II})$. The solid curves represent fits using the EOS form $\mathrm{HO2}$ for $\mathrm{Sc}(\mathrm{I})$ and $H 11$ for $\mathrm{Sc}(\mathrm{II})$ as discussed in the text. The mixedphase region of $\mathrm{Sc}(\mathrm{I})$ and $\mathrm{Sc}(\mathrm{II})$ is denoted by the hatched area.

$p=3 K_{0}(1-x) / x^{5} \exp \left[(3 / 2)\left(K_{0}^{\prime}-3\right)\right]$ with $x=\left(V / V_{0}\right)^{1 / 3}$ and in a second step the closely related first-order form ${ }^{31} H 11$, where $K_{0}^{\prime}=3-\ln \left(3 K_{0} / p_{\mathrm{FG} 0}\right)$ with the Fermi-gas pressure $p_{\mathrm{FG} 0}=a_{F}\left(Z / V_{0}\right)^{5 / 3}$, the universal constant $a_{\mathrm{FG}}=0.02367$ $\mathrm{GPa} \mathrm{nm}{ }^{5}$, and the total electron density $Z / V_{0}$ for ambient pressure guarantees the correct asymptotic variation at strong compression. In fact, many "simple", solids" follow this first-order form $H 11$ over extremely wide ranges in pressure, and in the present case, the standard deviation of the fitted curve from the data even decreases slightly from $\sigma_{v}=0.653 \%$ to $0.643 \%$ when this additional constraint is introduced for $K_{0}^{\prime}$, leading to $V_{0}=0.035(10) \mathrm{nm}^{3}, K_{0}=5.8(70)$ $\mathrm{GPa}$, and the constrained value $K_{0}^{\prime}=5.7$, whereby the standard deviations of the fitted parameters reflect only the statistical uncertainties. Thus one should note that these param- eters serve only for the representation of the data, and the ability of the first-order form $H 11$ to fit the EOS data may indicate, that these data represent a rather regular behavior typical for "simple solids",31 in contrast to the data for the low-pressure phase $\mathrm{Sc}(\mathrm{I})$ with their unusually small value for $K_{0}^{\prime}$, typical for the anomalous region of the "regular', RE metals, which is also characterized by unusually small values of $K_{0}^{\prime}$ due to $s$ - $d$ electron transfer. ${ }^{4}$

\section{CONCLUSION}

The present EDXD data for Sc under pressures up to 75 GPa strongly support a different structural assignment for $\mathrm{Sc}$ (II) with respect to the previous results. ${ }^{8,9}$ The present data for $\mathrm{Sc}$ (II) fit to a body-centered-cubic unit cell with 12 atoms clustered around each lattice point. The intensity data give some hints for possible icosahedral clusters or perhaps somewhat distorted icosahedra with typical size of $(\sqrt{ } 3 / 4) a_{\mathrm{bcc}}$ and with some kind of low-symmetry orientation in this structure breaking the cubic symmetry of the unit cell and leading therefore to a "pseudo-cI24" structural assignment. Since the observed diffraction patterns and the corresponding structural assignments are rather unique with no previous example of this structure type for any element under any condition, more detailed high-pressure x-ray-diffraction measurements using image plates with synchrotron radiation are desirable to provide some further information on this special structure. In addition, the range of the stability for this unusual structure should be explored also for later theoretical modeling.

\section{ACKNOWLEDGMENTS}

This work was supported in parts by the Bundesministerium für Forschung und Technologie (BMFT) under Grant No. 055PPAXB through DESY VI. The authors would like to thank Professor K. A. Gschneidner, Jr., for providing the sample material, W. Sievers for technical assistance, and J. Otto for support at the EDXD facility in HASYLAB. Y.C.Z. is indebted to the Alexander von Humboldt Foundation for financial support.
*Present address: Gordon McKay Laboratory, Division of Applied Sciences, Harvard University, Cambridge, MA 02138.

${ }^{1}$ K. A. Gschneidner, Jr., J. Alloys Compounds 192, 1 (1993).

${ }^{2}$ A. Jayaraman and R. C. Sherwood, Phys. Rev. 134, A691 (1964).

${ }^{3}$ D. B. McWhan and A. L. Stevens, Phys. Rev. 139, A682 (1965).

${ }^{4}$ U. Benedict and W. B. Holzapfel, in Handbook on the Physics and Chemistry of Rare Earths, Vol. 17, edited by K. A. Gschneidner, Jr., L. Eyring, G. H. Lander, and G. R. Choppin (North-Holland, Amsterdam, 1993), p. 245.

${ }^{5}$ N. Hamaya, Y. Sakamoto, H. Fujihisa, Y. Fujii, K. T. Takemura, T. Kikegawa, and O. Shimomura, J. Phys. Condens. Matter 5, L369 (1993).

${ }^{6}$ G. J. Leigh, International Union of Pure and Applied Chemistry, Recommendations 1990 (Blackwell, Oxford, 1990).

${ }^{7}$ J. Wittig, C. Probst, F. A. Schmidt, and K. A. Gschneidner, Jr., Phys. Rev. Lett. 42, 469 (1979).

${ }^{8}$ Y. K. Vohra, W. A. Grosshans, and W. B. Holzapfel, Phys. Rev. B 25, 6019 (1982).
${ }^{9}$ J. Akella, J. Xu, and G. S. Smith, Physica 139\&140B, 285 (1986).

${ }^{10}$ P. Söderlind, Ph.D. thesis, Uppsala University, 1994.

${ }^{11}$ W. Petry, J. Trampenau, and C. Herzig, Phys. Rev. B 48, 881 (1993).

${ }^{12}$ H. Xia, G. Parthasarathy, H. Luo, Y. K. Vohra, and A. L. Ruoff, Phys. Rev. B 42, 6736 (1990).

${ }^{13}$ W. A. Grosshans, E.-F. Düsing, and W. B. Holzapfel, High Temp. High Pressure 16, 539 (1984).

${ }^{14}$ J. Otto, in HASYLAB Annual Reports 1993 (DESY, Hamburg, 1994), p. 931.

${ }^{15}$ K. Syassen and W. B. Holzapfel, Europhys. Conf. Abstr. 1A, 75 (1975).

${ }^{16}$ W. B. Holzapfel, in High Pressure Chemistry, edited by H. Kelm (Reidel, Boston, 1978), p. 177.

${ }^{17}$ H. K. Mao, P. M. Bell, J. W. Shaner, and D. J. Steinberg, J. Appl. Phys. 49, 3276 (1978).

${ }^{18}$ F. Porsch (unpublished). 
${ }^{19}$ D. A. Young, Phase Diagrams of the Elements (University of California Press, Berkeley, 1990).

${ }^{20}$ Y. C. Zhao, F. Porsch, and W. B. Holzapfel, Phys. Rev. B 49, 815 (1994).

${ }^{21}$ M. Winzenick, V. Vijayakumar, and W. B. Holzapfel, Phys. Rev. B 50, 12381 (1994).

${ }^{22}$ W. B. Holzapfel, J. Alloys Compounds 223, 170 (1995).

${ }^{23}$ W. B. Holzapfel, Rep. Prog. Phys. 59, 39 (1996).

${ }^{24}$ M. Hoare, Ann. N. Y. Acad. Sci. 279, 186 (1976)

${ }^{25}$ E. N. Maslen, A. G. Fox, and M. A. O'Keefe, in International Tables for Crystallography, edited by A. J. C. Wilson (Kluwer, Dordrecht, 1992), Vol. C, p. 476.
${ }^{26}$ G. Venkataraman, D. Sahoo, and V. Balakrishnan, Beyond the Crystalline State (Springer-Verlag, Berlin, 1989).

${ }^{27}$ B. Buras and L. Gerward, Prog. Cryst. Growth Charact. 18, 93 (1989).

${ }^{28}$ W. A. Grosshans, Ph.D. thesis, University of Paderborn, 1987.

${ }^{29}$ W. A. Grosshans and W. B. Holzapfel, Phys. Rev. B 45, 5171 (1992).

${ }^{30}$ W. B. Holzapfel, in Molecular Systems under High Pressures, edited by R. Pucci and G. Piccitto (North-Holland, Amsterdam, 1991).

${ }^{31}$ W. B. Holzapfel, Europhys. Lett. 16, 67 (1991). 